\title{
Holographic multicondensate with nonlinear terms
}

\author{
Xing-Kun Zhang, Chuan-Yin Xia, Zhang-Yu Nie๑, , and Hui Zeng ${ }^{\dagger}$ \\ Kunming University of Science and Technology, Kunming 650500, China
}

(Received 26 September 2021; accepted 31 January 2022; published 25 February 2022)

\begin{abstract}
We study the influence of nonlinear terms quartic of the condensates on the phase structure of a holographic model with a multicondensate in the probe limit. We include one s-wave order and one p-wave order charged under the same U(1) gauge field in the holographic model and study the influence of the three quartic nonlinear terms with coefficients $\lambda_{s}, \lambda_{p}$ and $\lambda_{s p}$ on the phase structure. The quartic terms in action result in cubic nonlinear terms in equations of motion, which do not affect the critical points of a single condensate because the infinitesimal condensate value vanishes the nonlinear contributions. However, the quartic terms have a clear influence on the phase structure of systems containing multicondensates. We show the influence of each of the three parameters on the phase diagram with the other two set to zero, respectively. With these nonlinear terms, we get new power on tuning the phase structure of the holographic systems showing multicondensates, and realize a reentrant phase transition as an example.
\end{abstract}

DOI: 10.1103/PhysRevD.105.046016

\section{INTRODUCTION}

The holographic superconductor models with a single condensate [1-5] have been extended to systems with multiple orders [6] in recent years. The competition and coexistence of two orders are first studied in a holographic $\mathrm{s}+\mathrm{s}$ model [7,8], and later in $\mathrm{s}+\mathrm{p}$ [9-12] and $\mathrm{s}+\mathrm{d}$ $[13,14]$ models. In these models, besides the single condensate solutions, new coexistent solutions also exist; as a result, the holographic models with a multicondensate show rich phase transitions and the phase structures become more complicated.

With a multicondensates, the holographic models permit novel phase transitions even in the probe limit, which is quite useful for further studies involving time evolution. For example, in Ref. [15], phase separation is realized in a rotating system with two s-wave condensates holographically in the probe limit. In Ref. [16], the authors use a similar model with two s-wave orders to get a first order phase transition in the probe limit, and show the formation of two-dimensional bubble structure in first order phase transitions holographically. In the holographic study of time dependent physics [17-21], taking the probe limit

\footnotetext{
${ }^{*}$ Corresponding author. niezy@kust.edu.cn

Corresponding author. zenghui@kust.edu.cn

Published by the American Physical Society under the terms of the Creative Commons Attribution 4.0 International license. Further distribution of this work must maintain attribution to the author(s) and the published article's title, journal citation, and DOI. Funded by SCOAP ${ }^{3}$.
}

helps avoid the complicated numerical relativity problems. Therefore, exploring the phase structure of the holographic models with multiple condensates in the probe limit is important and benefits future studies.

In the study of the phase structure of the holographic models, the influence of various parameters on the critical points is quite crucial. The quartic nonlinear terms such as $\lambda|\Psi|^{4}$ may change the phase transition from a second order one to a first order one [22], but do not change position of the critical point which is determined by the physics in the linear region. However, in systems with a multiple condensate, the critical points of the coexistent solution are emerging from the single condensate solution with finite condensate value; as a result, the phase structure involving the coexistent solution gets nontrivial effects from such nonlinear terms. Therefore, it is interesting to study the influence of the quartic nonlinear terms on the phase structure of multicondensate holographic models and summarize possible universality.

It is difficult to build holographic models dual to a special material, therefore recent studies mainly focus on possible universality in the strongly coupled systems, where a first step is usually realizing specific phase transitions and various phase diagrams [23-25]. One kind of interesting phase transition is the reentrant one, which exhibits nonmonotonic dependence of the order parameter, and has already been realized holographically. However, the reentrant phase transition is usually studied in a holographic model with considering backreaction on the metric [10], or involving physical problems such as causality violation [26], which block further investigations. With the nonlinear parameters turned on, we get new power on tuning the phase transitions, because these nonlinear terms 
have a different influence than that of the other parameters such as charge $q$ and mass $m^{2}$, which change the thermodynamic potential curve "parallel" [26].

In this paper, we study the influence of three quartic nonlinear terms on the phase structure of a holographic $\mathrm{s}+\mathrm{p}$ model in the probe limit, and build a reentrant phase transition to test the power of tuning the phase transitions. In Sec. II we give the basic setup of the holographic model, and show details of calculation. In Sec. III, we show how the quartic terms influence the phase structure of the holographic system with a multicondensate, and show how to use the new power to get a reentrant phase transition [27] as an example. We conclude and discuss our results and some future topics in Sec. IV.

\section{HOLOGRAPHIC MODEL OF AN S + P SUPERCONDUCTOR}

\section{A. The model setup}

We consider a holographic model with one s-wave order and one $\mathrm{p}$-wave order in $(3+1)$-dimensional asymptotic AdS spacetime. The action of this holographic $s+$ p model is

$$
\begin{gathered}
S=S_{M}+S_{G}, \\
S_{G}=\frac{1}{2 \kappa_{g}^{2}} \int d^{4} x \sqrt{-g}(R-2 \Lambda), \\
S_{M}=\int d^{4} x \sqrt{-g}\left(-\frac{1}{4} \tilde{F}_{\mu \nu} \tilde{F}^{\mu \nu}-D_{\mu} \tilde{\psi}^{*} D^{\mu} \tilde{\psi}-m_{s}^{2} \tilde{\psi}^{*} \tilde{\psi}\right. \\
-\frac{1}{2} \tilde{\rho}_{\mu \nu}^{\dagger} \tilde{\rho}^{\mu \nu}-m_{p}^{2} \tilde{\rho}_{\mu}^{\dagger} \tilde{\rho}^{\mu} \\
\left.-\tilde{\lambda}_{s}\left(\tilde{\psi}^{*} \tilde{\psi}\right)^{2}-\tilde{\lambda}_{p}\left(\tilde{\rho}_{\mu}^{\dagger} \tilde{\rho}^{\mu}\right)^{2}-\tilde{\lambda}_{s p} \tilde{\psi}^{*} \tilde{\psi} \tilde{\rho}_{\mu}^{\dagger} \tilde{\rho}^{\mu}\right),
\end{gathered}
$$

where $\tilde{F}_{\mu \nu}=\nabla_{\mu} \tilde{A}_{\nu}-\nabla_{\nu} \tilde{A}_{\mu}$ is the Maxwell field strength and $D_{\mu} \tilde{\psi}=\nabla_{\mu} \tilde{\psi}-i \tilde{q}_{s} \tilde{A}_{\mu} \tilde{\psi} . \tilde{\rho}_{\mu \nu}=\bar{D}_{\mu} \tilde{\rho}_{\mu}-\bar{D}_{\nu} \tilde{\rho}_{\mu}$ is the field strength of the vector fields $\tilde{\rho}_{\mu}$, with the covariant derivatives $\bar{D}_{\mu}=\nabla_{\mu}-i \tilde{q}_{p} \tilde{A}_{\mu} \cdot \Lambda=-3 / L^{2}$ is the negative cosmological constant and $L$ is the AdS radius.

For simplicity, we work in the probe limit where the contribution of matter action $S_{M}$ is sufficiently small compared to the gravity action $S_{G}$. In such a limit, the spacetime background is not affected by the matter fields, therefore can be fixed to be the $(3+1)$-dimensional black brane with metric

$$
d s^{2}=-f(r) d t^{2}+\frac{1}{f(r)} d r^{2}+r^{2} d x^{2}+r^{2} d y^{2},
$$

where the function $f(r)$ is given by

$$
f(r)=\frac{r^{2}}{L^{2}}\left(1-\left(\frac{r_{h}}{r}\right)^{3}\right),
$$

with $r_{h}$ the horizon radius. The Hawking temperature of this black brane solution is

$$
T=\frac{3 r_{h}}{4 \pi L^{2}} .
$$

To approach the probe limit consistently, the matter fields in Eq. (3) are required to be infinitesimal. However, it is convenient to rescale the matter fields to be finite, using the following scaling symmetry of the system:

$$
\begin{array}{rlrl}
\tilde{A}_{\mu} & \rightarrow \lambda A_{\mu}, & \tilde{\psi} & \rightarrow \lambda \psi, \quad \tilde{\rho}_{\mu} \rightarrow \lambda \rho_{\mu}, \\
\tilde{q}_{s} & \rightarrow \lambda^{-1} q_{s}, & \tilde{q}_{p} & \rightarrow \lambda^{-1} q_{p}, \\
\tilde{\lambda}_{s} & \rightarrow \lambda^{-2} \lambda_{s}, & \tilde{\lambda}_{p} \rightarrow \lambda^{-2} \lambda_{p}, \quad \tilde{\lambda}_{s p} \rightarrow \lambda^{-2} \lambda_{s p} .
\end{array}
$$

Applying this scaling symmetry, only an overall factor $\lambda^{2}$ appears before the new form of matter action. We further choose the value of $\lambda$ to be the old value of $\tilde{q}_{s}^{-1}$ in Eq. (3), and the matter action becomes

$$
\begin{aligned}
S_{M}= & \frac{1}{\tilde{q}_{s}^{2}} \int d^{4} x \sqrt{-g}\left(-\frac{1}{4} F_{\mu \nu} F^{\mu \nu}-D_{\mu} \psi^{*} D^{\mu} \psi\right. \\
& -m_{s}^{2} \psi^{*} \psi-\frac{1}{2} \rho_{\mu \nu}^{\dagger} \rho^{\mu \nu}-m_{p}^{2} \rho_{\mu}^{\dagger} \rho^{\mu} \\
& \left.-\lambda_{s}\left(\psi^{*} \psi\right)^{2}-\lambda_{p}\left(\rho_{\mu}^{\dagger} \rho^{\mu}\right)^{2}-\lambda_{s p} \psi^{*} \psi \rho_{\mu}^{\dagger} \rho^{\mu}\right),
\end{aligned}
$$

where

$$
\begin{gathered}
F_{\mu \nu}=\nabla_{\mu} A_{\nu}-\nabla_{\nu} A_{\mu}, \\
\rho_{\mu \nu}=\bar{D}_{\mu} \rho_{\mu}-\bar{D}_{\nu} \rho_{\mu},
\end{gathered}
$$

with the new covariant derivatives

$$
\begin{gathered}
D_{\mu}=\nabla_{\mu}-i A_{\mu}, \\
\bar{D}_{\mu}=\nabla_{\mu}-i q_{p} A_{\mu} .
\end{gathered}
$$

In the new covariant derivatives, the new value of $q_{s}$ is equal to 1 , while the new value of $q_{p}$ is equal to the old value of the ratio $\tilde{q}_{p} / \tilde{q}_{s}$. It is obvious that with a sufficiently large value of $\tilde{q}_{s}$ (or in the limit $\tilde{q}_{s} \rightarrow \infty$ ), the finite valued configuration of the matter fields in Eq. (8) does not affect the metric, which is a consistent approach to the probe limit. It is also obvious that the quartic terms in the new form of matter action (8) make a finite contribution to the matter equations of motion, which corresponds to sufficiently large values of the coupling coefficients $\tilde{\lambda}_{s}, \tilde{\lambda}_{p}$ and $\tilde{\lambda}_{s p}$ in the old version of matter action (3).

We continue the rest of this work in the probe limit consistently with the new form of matter action (8). The consistent ansatz for the matter fields can be taken as 


$$
\psi=\psi_{s}(r), \quad A_{t}=\phi(r), \quad \rho_{x}=\psi_{p}(r),
$$

where all other field components are set to zero. Consequently, the equations of motion of matter fields are

$$
\begin{gathered}
\phi^{\prime \prime}+\frac{2}{r} \phi^{\prime}-2\left(\frac{\psi_{s}^{2}}{f}+\frac{q_{p}^{2} L^{2} \psi_{p}^{2}}{q_{s}^{2} r^{2} f}\right) \phi=0, \\
\psi_{p}^{\prime \prime}+\frac{f^{\prime}}{f} \psi_{p}^{\prime}+\left(\frac{q_{p}^{2} \phi^{2}}{q_{s}^{2} f^{2}}-\frac{m_{p}^{2}}{f}-\frac{\lambda_{s p} \psi_{s}^{2}}{f}\right) \psi_{p}-\frac{2 \lambda_{p} L^{2}}{r^{2} f} \psi_{p}^{3}=0,
\end{gathered}
$$

$$
\begin{aligned}
\psi_{s}^{\prime \prime} & +\left(\frac{f^{\prime}}{f}+\frac{2}{r}\right) \psi_{s}^{\prime}+\left(\frac{\phi^{2}}{f^{2}}-\frac{m_{s}^{2}}{f}-\frac{\lambda_{s p} L^{2} \psi_{p}^{2}}{r^{2} f}\right) \psi_{s} \\
- & \frac{2 \lambda_{s}}{f} \psi_{s}^{3}=0 .
\end{aligned}
$$

The three terms in the action $-\lambda_{s}\left(\psi^{*} \psi\right)^{2},-\lambda_{p}\left(\rho_{\mu}^{\dagger} \rho^{\mu}\right)^{2}$, $-\lambda_{s p} \psi^{*} \psi \rho_{\mu}^{\dagger} \rho^{\mu}$ are quartic to the charged fields $\psi_{s}$ and $\psi_{p}$ and introduce nonlinear cubic terms to the two equations (15) and (16). The nonlinear property of the terms in the single equation is important to understand the change of the critical points. Therefore we call these terms nonlinear in the sense that the single equation (15) or (16) is not linear on the condensate fields $\psi_{p}$ and $\psi_{s}$, although the equations of motion are already not linear as a whole even without these quartic terms.

\section{B. Boundary conditions}

To solve the equations of motion, we need boundary conditions both on the horizon and on the boundary. The boundary expansions of the three fields on the horizon side are

$$
\begin{gathered}
\phi(r)=\phi_{1}\left(r-r_{h}\right)+\mathcal{O}\left(\left(r-r_{h}\right)^{2}\right), \\
\psi_{s}(r)=\psi_{s 0}+\psi_{s 1}\left(r-r_{h}\right)+\mathcal{O}\left(r-r_{h}\right), \\
\psi_{p}(r)=\psi_{p 0}+\psi_{p 1}\left(r-r_{h}\right)+\mathcal{O}\left(r-r_{h}\right),
\end{gathered}
$$

where $\phi\left(r=r_{h}\right)$ is set to zero to meet the physical constraints from the finite norm of $A_{\mu}$ and the equations of motion (15) and (16) imply additional constraints on $\psi_{s 1}$ and $\psi_{p 1}$. Therefore, only $\phi_{1}, \psi_{s 0}, \psi_{p 0}$ are independent parameters. The expansions near the AdS boundary are

$$
\begin{gathered}
\phi(r)=\mu-\frac{\rho_{c}}{r}+\cdots, \\
\psi_{p}=\frac{\psi_{p_{-}}}{r^{\Delta_{p-}}}+\frac{\psi_{p_{+}}}{r^{\Delta_{p+}}}+\cdots, \\
\psi_{s}=\frac{\psi_{s_{-}}}{r^{\Delta_{s-}}}+\frac{\psi_{s_{+}}}{r^{\Delta_{s+}}}+\cdots,
\end{gathered}
$$

where

$$
\Delta_{s \pm}=\frac{\left(3 \pm \sqrt{9+4 m_{s}^{2}}\right)}{2}, \quad \Delta_{p \pm}=\frac{\left(1 \pm \sqrt{1+4 m_{p}^{2}}\right)}{2} .
$$

$\mu$ and $\rho_{c}$ are the chemical potential and charge density of the boundary field theory, respectively. We choose standard quantization, which means that $\psi_{s-}$ and $\psi_{p-}$ are the sources of the s-wave and p-wave orders, while $\psi_{s+}$ and $\psi_{p+}$ are the expectation values. The 3 degrees of freedom are fixed with $\psi_{s-}=\psi_{p-}=0$ and the value of $\mu$.

\section{Grand potential}

In order to compare the stability of the different solutions and get the final phase diagram, we study in the grand canonical ensemble and calculate the grand potential to find the stability relation between different solutions. The grand potential of the system can be identified with the temperature times the Euclidean on-shell action of the bulk solution according to the AdS/CFT correspondence. In this paper we work in the probe limit, therefore, the difference of the grand potential only comes from the matter part of the action,

$$
\Omega_{m}=T S_{M E},
$$

where $T$ is the Hawking temperature and $S_{M E}$ is the value of the on-shell matter action calculated with Euclidean time. $\Omega_{m}$ is the contribution from the matter part to the grand potential. With the equations of motion substituted in, $\Omega_{m}$ can be expressed as

$$
\begin{aligned}
\Omega_{m}= & V_{2}\left(-\frac{\mu \rho}{2 L^{2}}+\int_{r_{h}}^{\infty}\left(\frac{q_{s}^{2} r^{2} \phi^{2} \psi_{s}^{2}}{L^{2} f}+\frac{q_{p}^{2} \phi^{2} \psi_{p}^{2}}{f}\right.\right. \\
& \left.\left.-\frac{\lambda_{s} r^{2} \psi_{s}^{4}}{L^{2}}-\frac{\lambda_{p} L^{2} \psi_{p}^{4}}{r^{2}}-\lambda_{s p} \psi_{s}^{2} \psi_{p}^{2}\right) d r\right) .
\end{aligned}
$$

\section{The other two scaling symmetries}

Besides the scaling symmetry (7) we used to rescale the matter fields to define the probe limit, there are another two sets of scaling symmetries in Eqs. (14)-(16):

$$
\begin{aligned}
\phi & \rightarrow \lambda^{-2} \phi, \quad \psi_{s} \rightarrow \lambda^{-1} \psi_{s}, \quad f \rightarrow \lambda^{-2} f, \quad L \rightarrow \lambda^{-1} L, \\
m_{s}^{2} & \rightarrow \lambda^{-2} m_{s}^{2}, \quad m_{p}^{2} \rightarrow \lambda^{-2} m_{p}^{2} ; \\
\phi & \rightarrow \lambda^{-1} \phi, \quad \psi_{p} \rightarrow \lambda^{-1} \psi_{p}, \quad f \rightarrow \lambda^{-2} f, \\
r & \rightarrow \lambda^{-1} r, \quad r_{h} \rightarrow \lambda^{-1} r_{h} .
\end{aligned}
$$

With these two scaling symmetries, we set $r_{h}=L=1$ for numerical computation. After we get the numerical 
solutions, we can use again the two sets of scaling symmetries to recover $r_{h}$ and $L$ to any value.

It is worth noticing that with the scaling symmetry (7), $\tilde{q}_{s}$ or $\tilde{q}_{p}$ can be set to 1 without loss of generality in systems with single order. However, in this $\mathrm{s}+\mathrm{p}$ model, $\tilde{q}_{s}$ and $\tilde{q}_{p}$ are shifted simultaneously, and the ratio $\tilde{q}_{p} / \tilde{q}_{s}=q_{p}$ has a nontrivial effect on the final phase diagram. The change of $q_{p}$ shifts the grand potential curve of the p-wave solution parallel $[26,28]$, which is useful for tuning the phase transitions.

\section{INFLUENCE OF QUARTIC TERMS WITH PARAMETERS $\lambda_{s}, \lambda_{p}, \lambda_{s}$ ON THE PHASE DIAGRAM}

There are three different solutions with nonzero condensates, called the s-wave solution with nonzero condensate of the s-wave order, the p-wave solution with nonzero condensate of the p-wave order and the $\mathrm{s}+\mathrm{p}$ solution with both the two orders nonzero, respectively. It is easy to get the s-wave and p-wave solutions with a single condensate, while we need more technique to get the $s+p$ solution. It is conjectured that the $\mathrm{s}+\mathrm{p}$ solution always exists in a narrow region near the intersection point of the grand potential curves of s-wave and p-wave solutions in the probe limit [10]. Therefore, we need to locate this intersection point before we doing numerics to solve the $\mathrm{s}+\mathrm{p}$ solutions.

To focus on the influence of the three quartic terms with parameters $\lambda_{s}, \lambda_{p}$ and $\lambda_{s p}$, we first fix the value of the other parameters $m_{s}^{2}, m_{p}^{2}$ and $q_{s}, q_{p}$. In the study of time dependent evolution in holographic superconductors, it is wise to set the dimension $\Delta_{s}, \Delta_{p}$ to an integer value. A best choice for the mass parameters with $m_{s}^{2}=-2$ and $m_{p}^{2}=0$ further simplifies the equations [28].

We have to rescale the matter action to get finite valued matter fields in the probe limit, and set $q_{s}=1$ without loss of generality. Furthermore, we tune $q_{p}$ to make the grand potential of the s-wave solution and the p-wave solution have one intersection point when $\lambda_{s}=\lambda_{p}=\lambda_{s p}=0$, which indicates the existence of the coexistent solution. We should notice that, when we tune $q_{p}$, the grand potential curve for the p-wave solution moves parallel $[26,28]$.

Finally, we find a nice choice with $q_{p}=0.94239$ and $m_{s}^{2}=-2, m_{p}^{2}=0, q_{s}=1$. In the rest of this paper, we fix the values of the above parameters and focus on the influence of the three coefficients $\lambda_{s}, \lambda_{p}$ and $\lambda_{s p}$ of the nonlinear terms.

\section{A. Grand potential and condensates for $\lambda_{s}=\lambda_{p}=\lambda_{s p}=0$}

We start from the case with $\left(\lambda_{s}=\lambda_{p}=\lambda_{s p}=0\right)$ as a reference, where the grand potential curves of the s-wave solution and the p-wave solution have one intersection point. Near this intersection point, we can find the $\mathrm{s}+\mathrm{p}$ solution, which has the lowest value of grand potential. We
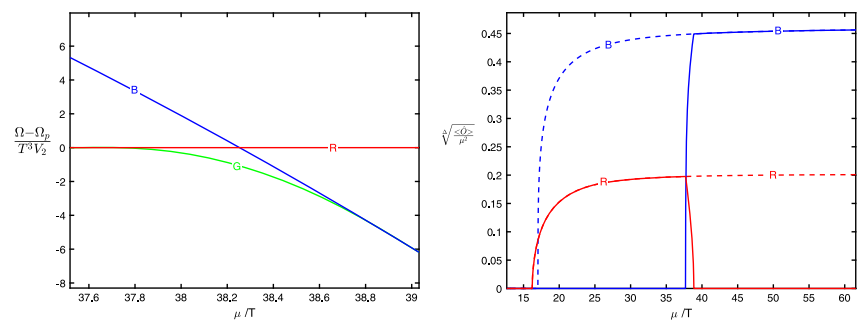

FIG. 1. Grand potential (left plot) and condensates (right plot) for different solutions. In the left plot, the three curves show the relative value of grand potential density with respect to the pwave solution $\left(\Omega-\Omega_{p}\right) / V_{2}$ for the s-wave solution (blue line marked with a letter B), the p-wave solution (red line marked with a letter R) and the $\mathrm{s}+\mathrm{p}$ solution (green line marked with a letter $\mathrm{G})$, respectively. In the right plot, the blue lines (marked with a letter B) and red lines (marked with a letter R) denote the condensate value for the s-wave and p-wave orders respectively. The solid lines show the condensate values for the most stable solutions while the dashed lines show the condensate values for the unstable region of the s-wave and p-wave solutions.

show the relative value of grand potential as well as the condensates in Fig. 1.

In the left plot of Fig. 1, we show the relative value of grand potential for different solutions with respect to the grand potential of the p-wave solution. We can see that with an increasing value of $\mu$, the $\mathrm{s}+\mathrm{p}$ solution emerges from the $\mathrm{p}$-wave solution, and then leaves away and merges with the s-wave solution finally. In the right plot, we show the condensate value of the two orders for different solutions. We can see that at the left critical point, the $s+p$ solution has an infinitesimal s-wave condensate, while at the right critical point, the $\mathrm{s}+\mathrm{p}$ solution has an infinitesimal $\mathrm{p}$-wave condensate. Therefore, in order to make the reference to the two critical points more clearly, we call the critical point with an infinitesimal s-wave condensate as the critical point for the s-wave order of the $\mathrm{s}+\mathrm{p}$ solution, and call the critical point with an infinitesimal $p$-wave condensate as the critical point for the $\mathrm{p}$-wave order of the $\mathrm{s}+\mathrm{p}$ solution. Both of the two critical points in Fig. 1 are second order, but the phase transitions may become first order when the three quartic terms are turned on. For convenience, when the phase transition becomes first order, we still use the word "critical point" to denote the spinodal point in first order phase transition, which also get an infinitesimal value of condensate for the relative order parameter.

\section{B. Influence of quartic terms with coefficients $\lambda_{s}$ and $\lambda_{p}$}

Based on the case with $\lambda_{s}=\lambda_{p}=\lambda_{s p}=0$ shown in the previous subsection, we further change $\lambda_{s}$ and $\lambda_{p}$ respectively to see the influence on the phase structure of the system. We also draw the $\lambda_{s}-\mu$ phase diagram with $\lambda_{p}=$ $\lambda_{s p}=0$ and the $\lambda_{p}-\mu$ phase diagram with $\lambda_{s}=\lambda_{s p}=0$ respectively in the end of this subsection to give a concrete view. 

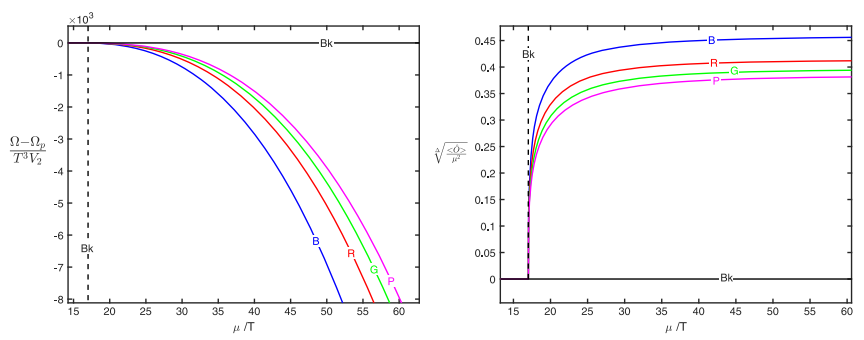

FIG. 2. The relative value of grand potential density for the swave solutions with respect to the normal solution (left plot) and the condensates of s-wave order for the s-wave solutions (right plot) with $\lambda_{s}=0$ (blue line marked with a letter B), $\lambda_{s}=0.5$ (red line marked with a letter R), $\lambda_{s}=1$ (green line marked with a letter $\mathrm{G}$ ) and $\lambda_{s}=1.5$ (purple line marked with a letter $\mathrm{P}$ ), respectively. The dashed black line marked with letters Bk represents the position of the critical point which is not influenced by the value of $\lambda_{s}$.

We first study the influence of the two parameters on the single condensate solutions. From the equations of motion, we can see that $\lambda_{s}$ does not change the $\mathrm{p}$-wave solution with $\psi_{s}=0$, and $\lambda_{p}$ does not change the s-wave solution with $\psi_{p}=0$ either. Therefore we can focus on the influence of $\lambda_{s}$ on the s-wave solution and the influence of $\lambda_{p}$ on the p-wave solution.

We fix $\lambda_{p}=0$ and show the grand potential as well as condensates of the s-wave solutions with different values of $\lambda_{s}$ in Fig. 2 . We can see that varying $\lambda_{s}$ does not change the critical point. This is because the term with $\lambda_{s}$ is nonlinear for $\psi_{s}$ in the single equation (16), and does not change the critical behavior determined by the infinitesimal value of $\psi_{s}$. Furthermore, it is obvious that the term with coefficient $\lambda_{s}$ has stronger influence on the s-wave solution with a larger value of condensate. This could also be explained by the cubic dependence on $\psi_{s}$ of the term $\lambda_{s} \psi_{s}^{3}$ in the equations of motion. The solution with a larger value of $\lambda_{s}$ get a higher value of grand potential, which indicates that the term $\lambda_{s} \psi_{s}^{4}$ is the potential of an effectively self-repulsive interaction.

The influence of the value of $\lambda_{p}$ on the p-wave solution with $\lambda_{s}=0$ is qualitatively the same to the influence of the value of $\lambda_{s}$ on the s-wave solution. We show the similar results of the grand potential as well as condensates for the p-wave solution with different values of $\lambda_{p}$ in Fig. 3.

Next, we study the influence of the two parameters on the coexistent $\mathrm{s}+\mathrm{p}$ solution. Again there are similarities between the influence of the values of $\lambda_{s}$ and $\lambda_{p}$, therefore we can focus on analysis of the case for $\lambda_{s}$, and the same analysis work in the case for $\lambda_{p}$.

We show the $\lambda_{s}-\mu$ phase diagram with $\lambda_{p}=\lambda_{s p}=0$ in the left plot of Fig. 4. In this plot, we use yellow, red, blue and green to denote the regions dominated by the normal phase, the $\mathrm{p}$-wave phase, the s-wave phase and the $\mathrm{s}+\mathrm{p}$ phase, respectively. The solid orange line between the
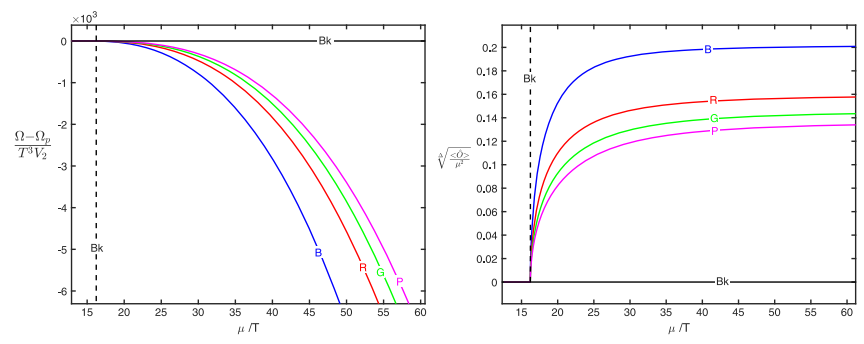

FIG. 3. The relative value of grand potential density for the pwave solutions with respect to the normal solution (left plot) and the condensates of $\mathrm{p}$-wave order for the p-wave solutions (right plot) with $\lambda_{p}=0$ (blue line marked with a letter B), $\lambda_{p}=0.5$ (red line marked with a letter $\mathrm{R}$ ), $\lambda_{p}=1$ (green line marked with a letter $\mathrm{G}$ ) and $\lambda_{s}=1.5$ (purple line marked with a letter P), respectively. The dashed black line marked with letters Bk represents the position of the critical point which is not influenced by the value of $\lambda_{p}$.

yellow and the red region presents the critical points of the p-wave solution and the dashed purple line denotes the critical points of the s-wave solution. The critical chemical potential for the $\mathrm{p}$-wave solution $\mu_{c-p}$ is always smaller than the critical chemical potential for the s-wave solution $\mu_{c-s}$, therefore the solid orange line is also a set of phase transition points while the dashed purple line is not. The blue and red solid lines represent the critical points for the $\mathrm{s}$-wave order and p-wave order of the $\mathrm{s}+\mathrm{p}$ solution, respectively. The dashed black line represents the intersection points of grand potential curves for the s-wave solution and the p-wave solution.

As we explained above, different values of $\lambda_{s}$ do not affect the critical points of the s-wave solution and p-wave
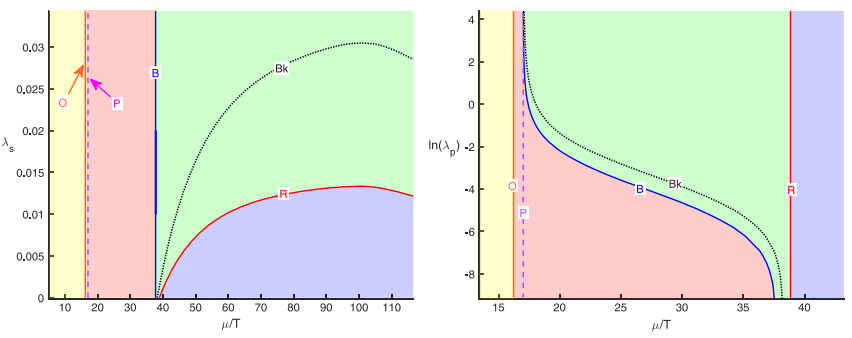

FIG. 4. The $\lambda_{s}-\mu$ phase diagram with $\lambda_{p}=\lambda_{s p}=0$ (left plot) and the $\lambda_{p}-\mu$ phase diagram with $\lambda_{s}=\lambda_{s p}=0$ (right plot). In the two phase diagrams, we use yellow, red, blue and green to denote the regions dominated by the normal phase, the $\mathrm{p}$-wave phase, the s-wave phase and the $s+p$ phase, respectively. The solid orange line (marked with a letter $\mathrm{O}$ ) between the yellow and the red region presents the critical points of the p-wave solution and the dashed purple line (marked with a letter P) denotes the critical points of the s-wave solution. The blue and red solid lines (marked with letters B and R respectively) represent the critical points for the s-wave order and p-wave order of the $\mathrm{s}+\mathrm{p}$ solution, respectively. The dashed black line (marked with letters $\mathrm{Bk}$ ) represents the intersection points of grand potential curves for the s-wave solution and the p-wave solution. 
solution, therefore both the solid orange and dashed purple curves are vertical. When the value of $\lambda_{s}$ becomes larger, the region of the $s+p$ phase increases as a result of the right critical point moving rightwards along the red solid line. However, the left critical point for the s-wave order does not move with the increasing of $\lambda_{s}$, and the blue solid line is also vertical. This property is also a result of infinitesimal condensate at critical points. It is obvious that the value of $\lambda_{s}$ does not change the p-wave solution with $\psi_{s}=0$. Furthermore, at the critical point for s-wave order of the $\mathrm{s}+\mathrm{p}$ solution, the s-wave condensate is infinitesimal, therefore this critical point is determined by the linearized version of Eq. (16) for $\psi_{s}$ with function $\phi$ determined by the p-wave solution. Both of the two factors are independent of $\lambda_{s}$, as a result, this critical point for s-wave order of the $\mathrm{s}+\mathrm{p}$ solution is not dependent on $\lambda_{s}$, and the solid blue line is vertical in the phase diagram.

Another property of the coexistent solution is that the red solid line denoting the critical point for $\mathrm{p}$-wave order of the $\mathrm{s}+\mathrm{p}$ solution is not monotonic and shows a maximum value of $\lambda_{s}=\lambda_{s}^{m}$. This implies that when $\lambda_{s}$ is slightly lower than $\lambda_{s}^{m}$, there are two segments for the $\mathrm{s}+\mathrm{p}$ solution. When $\lambda_{s}$ increases, both of the two segments of $\mathrm{s}+\mathrm{p}$ solution become wider, and the two critical points for $\mathrm{p}$-wave order join together at $\lambda_{s}=\lambda_{s}^{m}$. There should also exist a critical point for the s-wave order for the right segment of the $s+p$ solution. Because the critical point for the s-wave order would not be changed by different values of $\lambda_{s}$, this critical point should stay at $\mu=\infty$, just as the case with $\lambda_{s}=0$.

We can see that the dashed black line also shows a maximum value of $\lambda=\lambda_{s}^{I}$. This maximum implies that when $\lambda_{s}$ is slightly smaller than $\lambda_{s}^{I}$, there are two intersection points for the grand potential curves of the s-wave and p-wave solutions. According to the experience that the coexistent solution exits near the intersection point of the grand potential curves for single condensate solutions, the nonmonotonic property for the dotted black line is the reason for the nonmonotonic property for the solid red line.

We also draw the $\lambda_{p}-\mu$ phase diagram with $\lambda_{s}=\lambda_{s p}=0$ in the right plot of Fig. 4. In this plot, the solid orange line and dashed purple line are the same as in the left cousin. The influence of $\lambda_{p}$ on the phase structure is similar to the influence of $\lambda_{s}$, and the increasing of $\lambda_{p}$ enlarges the region of the $\mathrm{s}+\mathrm{p}$ phase. In contrast to the $\lambda_{s}-\mu$ phase diagram, when $\lambda_{p}$ increases, the left critical point for the s-wave order of the $\mathrm{s}+\mathrm{p}$ solution moves leftwards while the right critical point for the $\mathrm{p}$-wave order of the $\mathrm{s}+\mathrm{p}$ solution does not move and the solid red line is vertical. The same argument explains why the right critical point does not move with an increasing $\lambda_{p}$. The solid blue curve for the left critical point asymptotes to the dashed purple line, which might also be a result of the dashed black line asymptoting to the dashed purple line.

As the two quartic terms with coefficients $\lambda_{s}$ and $\lambda_{p}$ are potential terms in the Lagrangian, we focus on the positive values for $\lambda_{s}$ and $\lambda_{p}$ in this section. The minus values for the two parameters might cause runaway instability [22]. However, we show the two interesting phase diagrams with negative values of $\lambda_{s}$ and $\lambda_{p}$ in Appendix for possible interest.

\section{Influence of $\lambda_{s p}$ and the first order phase transition}

The influence of an interaction term between the two condensates, such as the one with coefficient $\lambda_{s p}$ in this paper, has already been studied in previous work in the holographic $\mathrm{s}+\mathrm{d}$ model [13] and the holographic $\mathrm{s}+\mathrm{s}$ model [16] respectively. The same as the results in these previous studies, the term with $\lambda_{s p}$ does not change the single condensate solutions, therefore we focus on the small region near the tricritical point. We show the $\lambda_{s p}-\mu$ phase diagram with $\lambda_{s}=\lambda_{p}=0$ for this model in Fig. 5. In this plot, we use blue, red and green to denote the region dominated by the s-wave phase, the p-wave phase and the $\mathrm{s}+\mathrm{p}$ phase, respectively. The blue and red lines represent the critical points for the s-wave and p-wave orders of the $\mathrm{s}+\mathrm{p}$ phase, respectively. The solid section of the two lines is also second order phase transition points, while the dashed lines are not real phase transition points, because the phase transitions become first order. The solid black line represents the first order phase transition points between the s-wave phase and the p-wave phase, while the solid green line represents the first order phase transition points between the $\mathrm{p}$-wave phase and the $\mathrm{s}+\mathrm{p}$ phase.

When $\lambda_{s p}$ is increasing, the two critical points moving towards each other along the red and blue lines, making the coexistent phase shrinking and finally disappearing at a

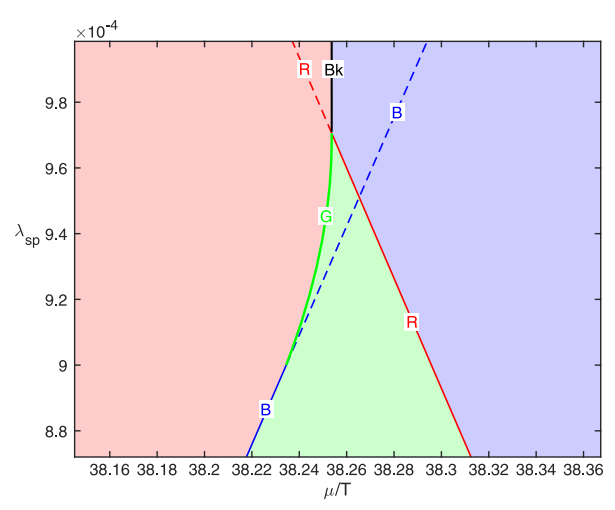

FIG. 5. The $\lambda_{s p}-\mu$ phase diagram with $\lambda_{s}=\lambda_{p}=0$. The blue, red and green regions are dominated by the s-wave phase, the pwave phase and the coexistent $s+p$ phase, respectively. The blue line (marked with a letter B) represents the critical points for the $\mathrm{s}$-wave order of the $\mathrm{s}+\mathrm{p}$ solution, the red line (marked with a letter R) represents the critical points for the $\mathrm{p}$-wave order of the $\mathrm{s}+\mathrm{p}$ solution. The solid black line (marked with letters Bk) represents the first order phase transition points between the $\mathrm{s}$-wave phase and the p-wave phase, while the solid green line (marked with a letter $\mathrm{G}$ ) represents the first order phase transition points between the $\mathrm{p}$-wave phase and the $\mathrm{s}+\mathrm{p}$ phase. 
tricritical point with $\lambda_{c} \approx 0.00097$. After $\lambda_{s p}$ reaches the tricritical point and goes on increasing, the $\mathrm{s}+\mathrm{p}$ solution is totally unstable and leaves a first order phase transition between the s-wave phase and the p-wave phase. With increasing of $\lambda_{s p}$, the two critical points for the $\mathrm{s}+\mathrm{p}$ solution keep moving along the dashed lines and the unstable $\mathrm{s}+\mathrm{p}$ solution becomes wider. It turns out that the term with parameter $\lambda_{s p}$ produces an effectively repulsive interaction between the two condensates.

Note that the intersection point of the blue and red lines is not on the black line. As a result, the phase transition from the $\mathrm{p}$-wave phase to the $\mathrm{s}+\mathrm{p}$ phase becomes first order near the tricritical point. This is qualitatively the same as the phase diagram in Ref. [16], but is different from that in Ref. [13], where the phase transition points between the single condensate phases and the coexistent phase are always second order. We believe that the detailed small section of first order phase transition is omitted in this earliest result on the topic [13] because of the sparse numerical data points. The tricritical point without first order phase transition to the coexistent solution means a highly coincident flat plain in the grand potential landscape.

\section{Realizing a reentrant phase transition}

From the three phase diagrams, we are clear about the influence of three parameters $\lambda_{s}, \lambda_{p}$ and $\lambda_{s p}$. The clear influence of the three parameters gives us new power on tuning the phase transitions between the three condensate solutions. In this section, we study how to find a reentrant phase transition as a test of the new power.

Since we have fixed $m_{s}^{2}=-2, m_{p}^{2}=0$ and $q_{s}=1$, $q_{p}=0.94239$, we get the two values of critical chemical potential as $\mu_{s}=4.06$ for the s-wave solution and $\mu_{p}=$ 3.88 for the p-wave solution from the results in Fig. 1. Because the three parameters $\lambda_{s}, \lambda_{p}$ and $\lambda_{s p}$ do not change the critical points of the single condensate solutions, $\mu_{s}$ and $\mu_{p}$ would not change and we always have $\mu_{p}<\mu_{s}$. Therefore the system always takes a second order phase transition from normal phase to the $\mathrm{p}$-wave phase, and the grand potential curve of the p-wave solution is always lower than the s-wave solution on the small $\mu$ (left) side.

From the experience of previous studies, when the grand potential line for the p-wave solution is lower both on the left and right side, it is possible for the system to transform from the $\mathrm{p}$-wave solution to the $\mathrm{s}+\mathrm{p}$ solution at a small value of $\mu$ and reenter the $\mathrm{p}$-wave solution at a larger value of $\mu$ again. In the case with $\lambda_{s}=\lambda_{p}=\lambda_{s p}=0$, the grand potential curves of the two single condensate solutions have only one intersection point. Therefore we should tune some parameter to change the relation in the large $\mu$ (right) region. From the influence of $\lambda_{s}$ and $\lambda_{p}$ on the grand potential curves shown in Figs. 2 and 3, we can see that if we increase $\lambda_{s}$, the right side of the grand potential curve of the s-wave solution will be lifted up, therefore it is possible to make the grand potential curves of the s-wave and pwave solutions have two intersection points.

The dashed black line in the phase diagram in Fig. 4 shows the trace of the intersection point. We can see from the left plot of Fig. 4 that when $\lambda_{s}$ approaches the maximum value $\lambda_{s}^{I}$ for the dashed black curve, we get two intersection points for the two grand potential curves and the grand potential of the p-wave solution is lower than that of the swave solution both on the left and right sides as we want.

However, the $\lambda_{s}-\mu$ phase diagram shows that in the case of $\lambda_{s}$ approaches $\lambda_{s}^{I}$, the $\mathrm{s}+\mathrm{p}$ phase dominates the right side of the phase diagram, and we did not see the reentrance back to the $\mathrm{p}$-wave solution. At this time, let us see the $\lambda_{s p}-\mu$ phase diagram, and remember that increasing the value of $\lambda_{s p}$ reduces the width of the $\mathrm{s}+$ $\mathrm{p}$ solution. If we want to make the p-wave solution to be more stable than the $\mathrm{s}+\mathrm{p}$ solution in the right side, we just increase $\lambda_{s p}$. As a result, the two critical points for the $\mathrm{s}+\mathrm{p}$ solution will move towards each other and finally, we can get the reentrant phase transition. Another possible problem is that when we fix the value of $\lambda_{s}$ and increase $\lambda_{s p}$, the two critical points for the $\mathrm{p}$-wave order of $\mathrm{s}+\mathrm{p}$ solution may emerge from the center, and then the $\mathrm{s}+\mathrm{p}$ solution is divided into two segments. This problem can be easily solved by slightly increasing the value of $\lambda_{s}$, which makes the two critical points move towards each other and disappear after the two merge in the middle, as shown by the solid red curve in the left plot of Fig. 4 .

Finally, we get a very nice reentrant phase transition with $\lambda_{s}=0.035, \lambda_{s p}=0.0168$ and $m_{s}^{2}=-2, m_{p}^{2}=0, q_{s}=1$, $q_{p}=0.94239$, and we show the condensates in Fig. 6 . We can see in this case that if we increase the value of $\mu$ from the normal phase, the system first takes a phase transition from the normal phase to the $\mathrm{p}$-wave phase. Then it take the second phase transition from the $\mathrm{p}$-wave phase to

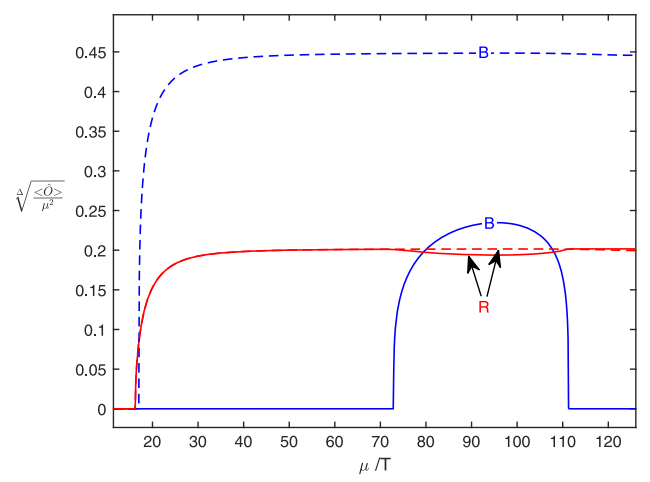

FIG. 6. Condensates of a reentrant phase transition with $m_{s}^{2}=-2, m_{p}^{2}=0, q_{s}=1, q_{p}=0.94239$ and $\lambda_{s}=0.035, \lambda_{p}=0$, $\lambda_{s p}=0.0168$. The red line (marked with a letter $\mathrm{R}$ ) denotes the condensate of the p-wave order while the blue line (marked with a letter B) denotes the condensate of the s-wave order. The solid lines are for the most stable solutions, while the dashed lines are for the unstable segments of the single condensate solutions. 
the $s+p$ phase. Finally, it takes the third phase transition from the $s+p$ phase back to the $p$-wave phase. All three phase transitions are second order, and the reentrance is realized accompanied by the nonmonotonic behavior for the s-wave order.

\section{CONCLUSION AND DISCUSSION}

In this paper, we consider the influence of three quartic nonlinear terms in the Lagrangian on the phase structure of a holographic $\mathrm{s}+\mathrm{p}$ model with one s-wave order and one p-wave order. We investigated the influence of the three parameters $\lambda_{s}, \lambda_{p}$ and $\lambda_{s p}$, respectively, and show the three phase diagrams. The influence of the quartic term similar to the term with coefficient $\lambda_{s p}$ in this paper has already been investigated in the $\mathrm{s}+\mathrm{d}$ model [13] as well as in the $\mathrm{s}+\mathrm{s}$ model [16]. Our study shows more similarity with the phase diagram in Ref. [16], which is more accurate than that in Ref. [13]. In addition, we studied the terms with coefficients $\lambda_{s}$ and $\lambda_{p}$. In the previous studies, only the $\lambda_{s}$ term is considered and been investigated near the critical point of the s-wave single condensate solutions [22]. We go beyond the critical region and show the influence of $\lambda_{s}$ and $\lambda_{p}$ terms on the condensates and grand potential density for single condensate solutions, and plot the resulting phase diagrams involving the coexistent phase. It is quite interesting that both of the two terms change one of the two critical points of coexistent solution while leaving the other critical point unchanged. This property is quite different with the $\lambda_{s p}$ term, and will enhance the power on engineering special phase transitions. With this new power on tuning the phase transitions, we successfully realized a reentrant phase transition as a test.

It should be noticed that, if we take two s-wave orders with the same best value of $m_{s}^{2}$, we are not expected to get the reentrant phase transition. This is because we can change from one s-wave solution with $q_{s}=q_{1}$ to the other s-wave solution with $q_{s}=q_{2}$ by using the scaling symmetry. Thus the two solutions with $\lambda_{s}=0$ are parallel to each other, and tuning $\lambda_{s}$ cannot make the grand potential curves having two intersection points. This is why we use an $\mathrm{s}+\mathrm{p}$ model instead of a more simpler $\mathrm{s}+\mathrm{s}$ model.

The influence of the $\lambda_{s p}$ term on the phase diagram is quite similar to those in $\mathrm{s}+\mathrm{s}$ and $\mathrm{s}+\mathrm{d}$ models. Although the $\lambda_{s}$ and $\lambda_{p}$ terms have not been investigated in other holographic models with a multicondensate, we expect similar qualitative results, such as the influence of the quartic terms on the grand potential of single condensate solutions, which is due to the repelling nature of the self-interaction potential terms with positive coefficients $\lambda_{s}$ and $\lambda_{p}$. Another interesting issue is that one of the critical points of the coexistent solution is not affected by $\lambda_{s}$ (or $\lambda_{p}$ ), which is not only observed in numerical results, but also clearly explained analytically in our study. The same nature of the quartic terms as well as the analytical explanation are also valid in $\mathrm{s}+\mathrm{s}, \mathrm{s}+\mathrm{d}$ and more general models. Therefore, we expect our results of the influence of the quartic terms nonlinear to the condensates to be qualitatively universal in other holographic models with multicondensates.

Our study may also shed light on real world systems dealing with multicondensates in condensed matter physics. For example, we can add similar nonlinear selfinteraction terms to change one of the critical points of the coexistent phase while the other critical point of the coexistent phase as well as the critical points for single condensate solutions are not changed. The new power on tuning phase transitions also provides a systematic and convenient way to get specific phase transitions, which is useful to investigate dynamical evolution and nonuniform structures of superfluids at strong coupling [16,28], where accurate tuning on critical points as well as grand potential relations becomes important. Moreover, special gravitational theories such as 4D and higher dimensional Einstein-Gauss-Bonnet gravity [29-31] may cause a special difference such as the influence on shear viscosity bound [32] and the nonmonotonic dependence of critical points on the Gauss-Bonnet coefficient [31], which is worth being studied with multicondensates in a future study.

\section{ACKNOWLEDGMENTS}

Z. Y. N. would like to thank Li Li for useful discussions. He would also like to thank the organizers of "2021 Xi' an Conference on Gravitation and Cosmology" for their hospitality. This work is partially supported by National Natural Science Foundation with Grants No. 11965013, No. 11565017. Z. Y. N. is partially supported by Yunnan Ten Thousand Talents Plan Young and Elite Talents Project.

\section{APPENDIX: THE PHASE DIAGRAMS WITH NEGATIVE VALUES OF $\lambda_{s}$ AND $\lambda_{p}$}

When the value of $\lambda_{s}$ (or $\lambda_{p}$ ) becomes negative, due to the contribution of the quartic term $-\lambda_{s} \psi_{s}^{4}$ (or $-\lambda_{p} \psi_{p}^{4}$ ), the holographic system will get a lower value of grand potential with a larger value of condensate. This implies that the system might be unstable because the grand potential is not bounded from below, which is also known as runaway pathology [22]. However, we can still get solutions with relatively small condensate value which is at least metastable and we show the related "phase diagrams" in this Appendix.

We extend the $\lambda_{s}-\mu$ phase diagram with $\lambda_{p}=\lambda_{s p}=0$ to negative values of $\lambda_{s}$ in Fig. 7 . We can see that the solid blue line is still vertical as we have explained, while the solid red line and the dashed black line go leftwards when the value of $\lambda_{s}$ decreases. Then the solid blue line, the solid red line and the dashed black line intersect near a small region. Near this small region, we can see that although the critical point denoted by the solid blue line does not move, when the phase transition becomes first order, the phase 

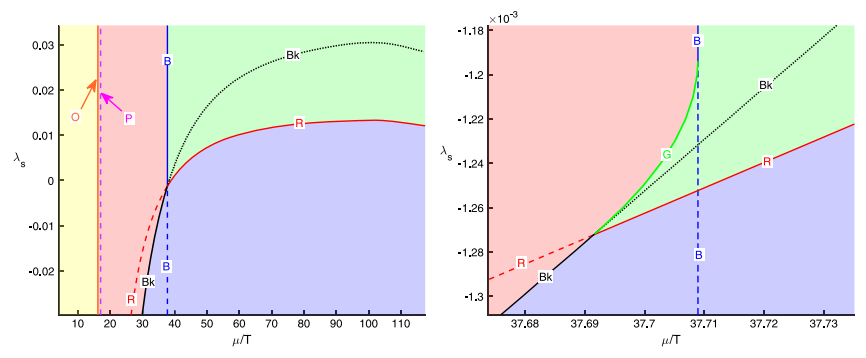

FIG. 7. The $\lambda_{s}-\mu$ phase diagram with $\lambda_{p}=\lambda_{s p}=0$ including the negative values of $\lambda_{s}$. The right plot is an enlarged version near the intersection points of the red, blue and black lines in the left plot. The solid green line (marked with a letter G) denotes the first order phase transition points between the p-wave phase and the $s+p$ phase, while the solid black line (marked with letters $\mathrm{Bk}$ ) denotes the first order phase transition points between the p-wave phase and the s-wave phase. The dashed parts of the blue and red lines (marked with letters $\mathrm{B}$ and $\mathrm{R}$ respectively) indicate that the critical points become spinodal points in first order phase transitions. The other notions are the same as that in the phase diagram with positive values of $\lambda_{s}$ in Fig. 4.

boundary between the red and green region moves leftwards along the solid green line. When the value of $\lambda_{s}$ is low enough, the region of $\mathrm{s}+\mathrm{p}$ phase disappears, and there is a first order phase transition between the s-wave phase and the p-wave phase marked by the solid black curve.

The case for the $\lambda_{p}-\mu$ phase diagram is similar. We also extend the $\lambda_{p}-\mu$ phase diagram with $\lambda_{s}=\lambda_{s p}=0$ to negative values of $\lambda_{p}$ in Fig. 8 . We can see that the solid red line is vertical, while the solid blue line and the dashed black line move rightwards when $\lambda_{p}$ decreases. The three lines also intersect in a small region. Near this small region, the phase transition from the $\mathrm{p}$-wave phase to the $\mathrm{s}+\mathrm{p}$ phase becomes first order and we use a solid green line to
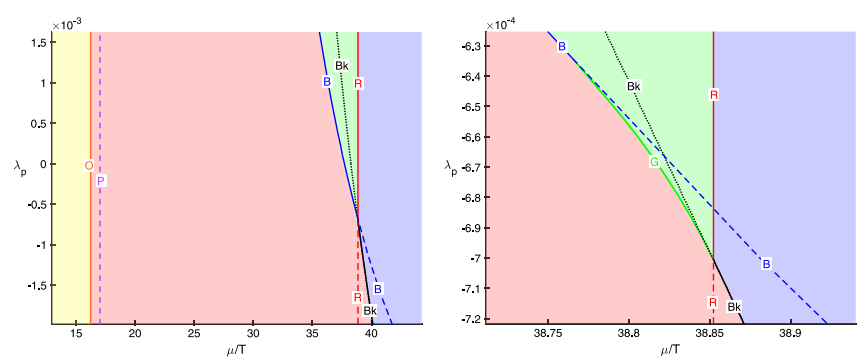

FIG. 8. The $\lambda_{p}-\mu$ phase diagram with $\lambda_{s}=\lambda_{s p}=0$ including the negative values of $\lambda_{p}$. The right plot is an enlarged version near the intersection points of the red, blue and black lines in the left plot. The solid green line (marked with a letter $G$ ) denotes the first order phase transition points between the $p$-wave phase and the $s+p$ phase, while the solid black line (marked with letters $\mathrm{Bk}$ ) denotes the first order phase transition points between the pwave phase and the s-wave phase. The dashed parts of the blue and red lines (marked with letters $\mathrm{B}$ and $\mathrm{R}$ respectively) indicate that the critical points become spinodal points in first order phase transitions. The other notions are the same as that in the phase diagram with positive values of $\lambda_{s}$ in Fig. 4 .

denote the phase transition points. Although the red line denoting the critical points for the $p$-wave order of the $s+p$ phase is always vertical, the $\mathrm{s}+\mathrm{p}$ phase disappears when $\lambda_{p}$ is low enough and there is a first order phase transition between the p-wave phase and the s-wave phase marked by the solid black curve.

In this paper, we focus on the phase diagram and reentrant phase transitions with positive values of $\lambda_{s}$ and $\lambda_{p}$ in the main text, which can be applied in further studies without worrying about the stability problem. The study on the instability of runaway pathologies [22] is also quite interesting, and we will further investigate the related problems in a future study.
[1] S. A. Hartnoll, C. P. Herzog, and G. T. Horowitz, Building a Holographic Superconductor, Phys. Rev. Lett. 101, 031601 (2008).

[2] S. S. Gubser and S. S. Pufu, The gravity dual of a $p$-wave superconductor, J. High Energy Phys. 11 (2008) 033.

[3] J. W. Chen, Y. J. Kao, D. Maity, W. Y. Wen, and C. P. Yeh, Towards a holographic model of D-wave superconductors, Phys. Rev. D 81, 106008 (2010).

[4] F. Benini, C. P. Herzog, R. Rahman, and A. Yarom, Gauge gravity duality for d-wave superconductors: Prospects and challenges, J. High Energy Phys. 11 (2010) 137.
[5] R. G. Cai, L. Li, and L. F. Li, A holographic $P$-wave superconductor model, J. High Energy Phys. 01 (2014) 032.

[6] R. G. Cai, L. Li, L. F. Li, and R. Q. Yang, Introduction to holographic superconductor models, Sci. China Phys. Mech. Astron. 58, 060401 (2015).

[7] P. Basu, J. He, A. Mukherjee, M. Rozali, and H.-H. Shieh, Competing holographic orders, J. High Energy Phys. 10 (2010) 092.

[8] R.-G. Cai, L. Li, L.-F. Li, and Y.-Q. Wang, Competition and coexistence of order parameters in holographic multi-band superconductors, J. High Energy Phys. 09 (2013) 074. 
[9] Z. Y. Nie, R. G. Cai, X. Gao, and H. Zeng, Competition between the s-wave and p-wave superconductivity phases in a holographic model, J. High Energy Phys. 11 (2013) 087.

[10] Z. Y. Nie, R. G. Cai, X. Gao, L. Li, and H. Zeng, Phase transitions in a holographic $\mathrm{s}+\mathrm{p}$ model with backreaction, Eur. Phys. J. C 75, 559 (2015).

[11] I. Amado, D. Arean, A. Jimenez-Alba, L. Melgar, and I. Salazar Landea, Holographic s + p superconductors, Phys. Rev. D 89, 026009 (2014).

[12] R. Arias and I. Salazar Landea, Spontaneous current in a holographic s + p superfluid, Phys. Rev. D 94, 126012 (2016).

[13] M. Nishida, Phase diagram of a holographic superconductor model with s-wave and d-wave, J. High Energy Phys. 09 (2014) 154.

[14] L. F. Li, R. G. Cai, L. Li, and Y. Q. Wang, Competition between s-wave order and d-wave order in holographic superconductors, J. High Energy Phys. 08 (2014) 164.

[15] W. C. Yang, C. Y. Xia, H. B. Zeng, and H. Q. Zhang, Phase separation and exotic vortex phases in a two-species holographic superfluid, Eur. Phys. J. C 81, 21 (2021).

[16] X. Li, Z. Y. Nie, and Y. Tian, Holographic boiling and generalized thermodynamic description beyond local equilibrium, J. High Energy Phys. 09 (2020) 063.

[17] M. J. Bhaseen, J. P. Gauntlett, B. D. Simons, J. Sonner, and T. Wiseman, Holographic Superfluids and the Dynamics of Symmetry Breaking, Phys. Rev. Lett. 110, 015301 (2013).

[18] A. Adams, P. M. Chesler, and H. Liu, Holographic vortex liquids and superfluid turbulence, Science 341, 368 (2013).

[19] A. Adams, P. M. Chesler, and H. Liu, Holographic Turbulence, Phys. Rev. Lett. 112, 151602 (2014).

[20] J. Sonner, A. del Campo, and W. H. Zurek, Universal farfrom-equilibrium dynamics of a holographic superconductor, Nat. Commun. 6, 7406 (2015).
[21] P. M. Chesler, A. M. Garcia-Garcia, and H. Liu, Defect Formation beyond Kibble-Zurek Mechanism and Holography, Phys. Rev. X 5, 021015 (2015).

[22] C. P. Herzog, An analytic holographic superconductor, Phys. Rev. D 81, 126009 (2010).

[23] Z. Y. Nie and H. Zeng, P-T phase diagram of a holographic $\mathrm{s}+\mathrm{p}$ model from Gauss-Bonnet gravity, J. High Energy Phys. 10 (2015) 047.

[24] E. Kiritsis and L. Li, Holographic competition of phases and superconductivity, J. High Energy Phys. 01 (2016) 147.

[25] J. W. Chen, S. H. Dai, D. Maity, and Y. L. Zhang, Engineering holographic phase diagrams, Phys. Rev. D 94, 086004 (2016).

[26] Z. H. Li, Y. C. Fu, and Z. Y. Nie, Competing s-wave orders from Einstein-Gauss-Bonnet gravity, Phys. Lett. B 776, 115 (2018).

[27] T. Narayanan and A. Kumar, Reentrant phase transitions in multicomponent liquid mixtures, Phys. Rep. 249, 135 (1994).

[28] C. Y. Xia, Z. Y. Nie, H. B. Zeng, and Y. Zhang, Uniform quenching processes in a holographic $\mathrm{s}+\mathrm{p}$ model with reentrance, Eur. Phys. J. C 81, 882 (2021).

[29] D. Glavan and C. Lin, Einstein-Gauss-Bonnet Gravity in Four-Dimensional Spacetime, Phys. Rev. Lett. 124, 081301 (2020).

[30] P. G. S. Fernandes, Charged black holes in AdS spaces in $4 D$ Einstein Gauss-Bonnet gravity, Phys. Lett. B 805, 135468 (2020).

[31] X. Qiao, L. OuYang, D. Wang, Q. Pan, and J. Jing, Holographic superconductors in 4D Einstein-Gauss-Bonnet gravity, J. High Energy Phys. 12 (2020) 192.

[32] M. Brigante, H. Liu, R. C. Myers, S. Shenker, and S. Yaida, The Viscosity Bound and Causality Violation, Phys. Rev. Lett. 100, 191601 (2008). 